\title{
Théorèmes d'Existence et d'Approximation pour les Équations aux Dérivées Partielles Linéaires d'Ordre Infini
}

\author{
Par \\ Ryuichi ISHIMURA
}

\section{§1. Introduction}

Dans le mémoire précédent [5], on a caractérisé l'homomorphisme continu $\mathrm{du}$ faisceau des germes de fonctions holomorphes dans lui-même comme un opérateur différentiel. Rappelons-nous ses résultats. Soient $\mathcal{O}$ le faisceau des germes de fonctions holomorphes sur $\mathbb{C}^{n}$ espace de $n$ variables complexes et $P: \mathcal{O} \rightarrow \mathcal{O}$ un homomorphisme de faisceaux. Pour chaque ouvert $\Omega$ de $\mathbb{C}^{n}$, l'espace $\Gamma(\Omega, \mathcal{O})$ des sections de $\mathcal{O}$ au-dessus de $\Omega$ muni de la topologie de la convergence compacte sur $\Omega$ est un espace de Fréchet. On dira que l'homomorphisme $P: \mathcal{O} \rightarrow \mathcal{O}$ est continu si pour chaque ouvert $\Omega$ de $\mathbb{C}^{n}$, l'application induite $P_{\Omega}: \Gamma(\Omega, \mathcal{O}) \rightarrow \Gamma(\Omega, \mathcal{O})$ est continue. On a démontré d'abord que l'homomorphisme continu $P: \mathcal{O} \rightarrow \mathcal{O}$ est un opérateur différentiel $p\left(z, \partial_{z}\right)$ dont l'ordre n'est pas nécessairement fini. Au symbole

$$
p(z, \zeta)=\sum_{\alpha} a_{\alpha}(z) \zeta^{\alpha}
$$

de l'opérateur différentiel $p\left(z, \partial_{z}\right)$, on associe la forme

$$
\tilde{p}(z, \zeta)=\sum_{\alpha} a_{\alpha}(z) \zeta^{\alpha} \alpha ! .
$$

On a établi les résultats suivants: Pour que l'homomorphisme $P$ de $\mathcal{O}$ dans $\mathcal{O}$ soit continu, il faut et il suffit que $P$ soit un opérateur différentiel sur $\mathbb{C}^{n}$ dont la forme $\tilde{p}(z, \zeta)$ est holomorphe dans $\mathbf{C}^{n} \times \mathbb{C}^{n}$.

Soient $p\left(\partial_{z}\right)$ un opérateur différentiel à coefficients constants sur $\mathbb{C}^{n}$ représenté par un homomorphisme continu $P$ de $\mathcal{O}$ dans $\mathcal{O}$ et $\Omega$ un ouvert de $\mathbb{C}^{n}$. On dit que l'ouvert $\Omega$ est pseudo $p\left(\partial_{z}\right)$-convexe si la condition suivante est

Communiqué par S. Matsuura, le 6 juillet, 1978.

* Département de Mathématiques, Faculté des Sciences, Université de Kyushu. 
vérifiée: si $T$ est une fonctionnelle analytique sur $\mathbf{C}^{n}$ telle que la fonctionnelle analytique ${ }^{t} P T$ soit appuyée par $\Omega, T$ est appuyée par $\Omega$ où ${ }^{t} P$ désigné la transposée de l'opérateur $P: \Gamma\left(\mathbf{C}^{n}, \mathcal{O}\right) \rightarrow \Gamma\left(\mathbf{C}^{n}, \mathcal{O}\right)$ induite par l'homomorphisme $P: \mathcal{O} \rightarrow \mathcal{O}$.

Dans ce mèmoire, on se propose de démontrer le théorème suivant:

Théorème. Soient $p\left(\partial_{z}\right)=\sum_{\alpha} a_{\alpha} \partial_{z}^{\alpha}$ un opérateur différentiel non nul à coefficients constants sur $\mathbf{C}^{n}$ représenté par un homomorphisme continu $P$ de $\mathcal{O}$ dans $\mathcal{O}$ et $\Omega$ un ouvert de type de Runge dans $\mathbf{C}^{n}$. Alors pour que les énoncés suivants (i) et (ii) soient valables, il faut et il suffit que l'ouvert $\Omega$ soit pseudo $p\left(\partial_{z}\right)$-convexe:

(i) Pour toute fonction holomorphe $f$ sur $\Omega$, il existe $u$ fonction holomorphe sur $\Omega$ tel que l'on ait:

$$
p\left(\partial_{z}\right) u=f
$$

dans $\Omega$.

(ii) Toute solution $u$ dans $\Gamma(\Omega, \mathcal{O})$ de l'équation homogène

$$
p\left(\partial_{z}\right) u=0
$$

est approchée dans $\Gamma(\Omega, \mathcal{O})$ par des exponentielle-polynômes satisfaisant à l'équation homogène.

On se propose de démontrer que tout ouvert convexe $\Omega$ est pseudo $p\left(\partial_{z}\right)$ convexe. C'est une généralisation du théorème 9.4 de Trèves [7] qui traite du cas où $p(\zeta)$ est un polynôme:

Théorème. Soient $p\left(\partial_{z}\right)=\sum_{\alpha} a_{\alpha} \partial_{z}^{\alpha}$ un opérateur différentiel non nul ̀̀ coefficients constants sur $\mathbf{C}^{n}$ représenté par un homomorphisme continu $P$ de $\mathcal{O}$ dans $\mathcal{O}$ et $\Omega$ un ouvert convexe de $\mathbf{C}^{n}$. Alors l'ouvert $\Omega$ est pseudo $p\left(\partial_{z}\right)$ convexe et l'on a donc les énoncés suivants (i) et (ii):

(i) Pour toute fonction holomorphe $f$ sur $\Omega$, il existe $u$ fonction holomorphe sur $\Omega$ tel que l'on ait:

$$
p\left(\partial_{z}\right) u=f
$$

dans $\Omega$.

(ii) Toute solution u dans $\Gamma(\Omega, \mathcal{O})$ de l'équation homogène

$$
p\left(\partial_{z}\right) u=0
$$


est approchée dans $\Gamma(\Omega, \mathcal{O})$ par des exponentielle-polynômes satisfaisant à l'équation homogène.

\section{§2. Notations et Rappels des Résultats Précédents}

Dans ce mémoire on désignera par $\mathcal{O}$ le faisceau des germes de fonctions holomorphes sur $\mathbf{C}^{n}$, par $\Gamma(\Omega, \mathcal{O})$ l'espace de Fréchet des sections de $\mathcal{O}$ au-dessus d'un ouvert $\Omega$ de $\mathbf{C}^{n}$ muni de la topologie de la convergence compacte sur $\Omega$ et par $N$ l'ensemble des entiers positifs. On utilisera les notations suivantes: pour tout $\alpha=\left(\alpha_{j}\right)_{1 \leqq j \leqq n} \in N^{n}$,

$$
\begin{aligned}
& |\alpha|=\alpha_{1}+\alpha_{2}+\cdots+\alpha_{n}, \\
& \alpha !=\alpha_{1} ! \alpha_{2} ! \cdots \alpha_{n} !, \\
& \partial_{z}^{\alpha}=\frac{\partial|\alpha|}{\partial_{z_{1}}^{\alpha_{1}} \partial_{z_{2}}^{\alpha_{2}} \cdots \partial_{z_{n}}^{\alpha_{n}}}
\end{aligned}
$$

et pour tout $\beta=\left(\beta_{j}\right)_{1 \leqq j \leqq n} \in N^{n}$ tel que $\beta \leqq \alpha$,

$$
\left(\begin{array}{l}
\alpha \\
\beta
\end{array}\right)=\left(\begin{array}{l}
\alpha_{1} \\
\beta_{1}
\end{array}\right)\left(\begin{array}{l}
\alpha_{2} \\
\beta_{2}
\end{array}\right) \cdots\left(\begin{array}{l}
\alpha_{n} \\
\beta_{n}
\end{array}\right) .
$$

Pour tout $z \in \mathbb{C}^{n}$ et tout nombre positif $r$, on désignera par $\Delta_{r}(z)$, le polydisque de $\mathbf{C}^{n}$ à centre $z$ et de rayon $r$. Pour tout compact $K$ de $\mathbf{C}^{n}$ et toute fonction $f$ sur $K$, on pose:

$$
\|f\|_{K}=\sup _{z \in \mathbb{R}}|f(z)| \text {. }
$$

Soient $E$ un espace vectoriel topologique et $E^{\prime}$ son dual topologique. Pour tout sous-espace $M$ de $E$, on définit $M^{0}$ sous-espace de $E^{\prime}$ par

$$
M^{0}=\left\{x^{\prime} \in E^{\prime} \mid x^{\prime}(x)=\left\langle x, x^{\prime}\right\rangle=0, \text { pour tout } x \in M\right\} .
$$

Définition 1. Soit $T: \Gamma\left(\mathbb{C}^{n}, \mathcal{O}\right) \rightarrow \Gamma\left(\mathbb{C}^{n}, \mathcal{O}\right)$ une application linéaire. On dira que $T$ est un opérateur différentiel sur $\mathbb{C}^{n}$ dont l'ordre n'est pas nécessairement fini si $T$ satisfait la condition suivante: il existe un unique $\left(a_{\alpha}\right)_{\alpha \in N^{n}}$ famille de fonctions holomorphes $a_{\alpha}$ sur $\mathbb{C}^{n}$ tel que pour tout ouvert $\Omega$ de $\mathbb{C}^{n}$ et toute fonction holomorphe $f$ sur $\Omega$, on ait:

$$
T f=\sum_{\alpha} a_{\alpha} \partial_{z}^{\alpha} f
$$

dans $\Omega$. 
Au symbole

$$
t(z, \zeta)=\sum_{\alpha} a_{\alpha}(z) \zeta^{\alpha}
$$

de l'opérateur différentiel $T$, on associe la forme

$$
\tilde{t}(z, \zeta)=\sum_{\alpha} a_{\alpha}(z) \zeta^{\alpha} \alpha ! .
$$

Pour touts $\alpha$ et $\beta$ de $N^{n}$, on utilisera la notation suivante:

$$
t_{(\beta)}^{(\alpha)}(z, \zeta)=\partial_{z}^{\beta} \partial_{\zeta}^{\alpha} t(z, \zeta)
$$

Soit $P: \mathcal{O} \rightarrow \mathcal{O}$ un homomorphisme de faisceaux. On dit que l'homomorphisme $P$ est continu si pour tout ouvert $\Omega$ de $\mathbf{C}^{n}$, l'application linéaire induite $P_{\Omega}: \Gamma(\Omega, \mathcal{O}) \rightarrow \Gamma(\Omega, \mathcal{O})$ est continue. Dans le mémoire précédent [5], on a établi le critère suivant:

Critère 1. Soit $P$ un homomorphisme continu de $\mathcal{O}$ dans $\mathcal{O}$. Alors $P$ est un opérateur différentiel sur $\mathbf{C}^{n}$ et la série $\tilde{p}(z, \zeta)$ associée à $P$ est holomorphe dans $\mathbf{C}^{n} \times \mathbf{C}^{n}$. Réciproquement, soit $P=\sum_{\alpha} a_{\alpha}(z) \partial_{z}^{\alpha}$ un opérateur différentiel sur $\mathbf{C}^{n}$ tel que la serie $\tilde{p}(z, \zeta)=\sum_{\alpha} a_{\alpha}(z) \zeta^{\alpha} \alpha !$ associée à $P$ soit holomorphe dans $\mathbf{C}^{n} \times \mathbf{C}^{n}$. Alors $P$ définit un homomorphisme continu de $\mathcal{O}$ dans $\mathcal{O}$.

\section{§3. Rappels des Résultats de Trèves}

D'après Trèves [8], on résumera les criterès des epimorphismes. Soient $E_{0}, F_{0}, E$ et $F$ quatre espaces localement convexes séparés et $u_{0}: E_{0} \rightarrow F_{0}, \rho$ : $E_{0} \rightarrow E$ et $\sigma: F_{0} \rightarrow F$ trois applications linéaires continues. On considérera le diagramme suivant:

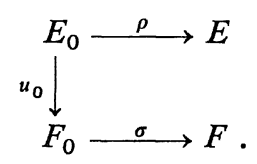

Définition 2. On dira que l'application $u_{0}: E_{0} \rightarrow F_{0}$ est essentiellement univalente par rapport au couple $(\rho, \sigma)$ si $u_{0}$ satisfait à la condition suivante: pour tout filtre $\mathfrak{F}$ dans $E_{0}$ tel que l'image $\rho(\mathfrak{F})$ converge dans $E$ à 0 et que l'image $\sigma \circ u_{0}(\mathfrak{F})$ converge dans $F$ à $y$ point de $F$, on a: $y=0$.

On définit $G_{0}$ sous-espace de l'espace vectoriel produit $E \times F$ comme suite: 


$$
G_{0}=\left\{\left(\rho\left(x_{0}\right), \sigma \circ u_{0}\left(x_{0}\right)\right) \mid x_{0} \in E_{0}\right\} .
$$

Soient $G$ l'adhérence de $G_{0}$ dans $E \times F$ et $\pi_{1}: E \times F \rightarrow E$ et $\pi_{2}: E \times F \rightarrow F$ les projections. On a le lemme suivant:

Lemme. Pour que l'application $u_{0}: E_{0} \rightarrow F_{0}$ soit essentiellement univalente, il faut et il suffit que la restriction $\pi_{1 \mid G}$ de $\pi_{1}$ à G soit injective.

D'après le lemme précédent, si l'application $u_{0}: E_{0} \rightarrow F_{0}$ est essentiellement univalente, la restriction $\pi_{1 \mid G}: G \rightarrow \widetilde{E}_{0}$ est bijective où $\widetilde{E}_{0}$ est l'image de $\pi_{1 \mid G}$ munie de la topologie induite par l'espace $E$. Soit $\tilde{u}_{0}: \widetilde{E}_{0} \rightarrow F$ le composé de l'application réciproque $\left(\pi_{1 \mid G}\right)^{-1}$ et la projection $\pi_{2}$. On appellera l'application $\tilde{u}_{0}$ l'extension canonique de $u_{0}$ par rapport au couple $(\rho, \sigma)$.

Définition 3. Soient l'application $u_{0}: E_{0} \rightarrow F_{0}$ essentiellement univalente et $\tilde{u}_{0}: \widetilde{E}_{0} \rightarrow F$ son extension canonique. On dit que $\tilde{u}_{0}$ a la properiété d'approximation homogène si l'espace $\rho\left(\operatorname{Ker} u_{0}\right)$ est dense dans $\operatorname{Ker} \tilde{u}_{0}$ sous-espace de E.

Dans la situation précédente, on a deux critères suivants:

Critère 2 (le corollaire 1 du théorème 17.2 de Trèves [8]). Soit l'application $u_{0}: E_{0} \rightarrow F_{0}$ essentiellement univalente. Supposons que $E$ et $F$ sont espaces de Fréchet, que la transposée ${ }^{t} u_{0}: F_{0}^{\prime} \rightarrow E_{0}^{\prime}$ de $u_{0}$ est injective et que l'on $a: \operatorname{Im}^{t} u_{0}=\left(\operatorname{Ker} u_{0}\right)^{0}$. Alors les propriétés suivantes sont équivalentes:

(a) L'extension canonique $\tilde{u}_{0}$ de $u_{0}$ est un epimorphisme de $\widetilde{E}_{0}$ sur $F$, ayant la propriété d'approximation homogène.

(b) L'espace Im $\sigma$ est dense dans $F$ et pour tout $y_{0}^{\prime} \in F_{0}^{\prime}$ tel que ${ }^{t} u_{0}\left(y_{0}^{\prime}\right) \in$ $\operatorname{Im}{ }^{t} \rho$, on $a: y_{0}^{\prime} \in \operatorname{Im}^{t} \sigma$.

Critère 3 (le corollaire 3 du théorème 17.2 de Trèves [8]). Soit l'application $u_{0}: E_{0} \rightarrow F_{0}$ essentiellement univalente. Supposons que $E_{0}, F_{0}, E$ et $F$ sont espaces de Fréchet et que $u_{0}: E_{0} \rightarrow F_{0}$ est surjective. Alors les propriétés suivantes sont équivalentes:

(a) L'extension canonique $\tilde{u}_{0}$ de $u_{0}$ est un epimorphisme de $\widetilde{E}_{0}$ sur $F$, ayant la propriété d'approximation homogène.

(b) L'espace Im $\sigma$ est dense dans $F$ et pour tout $y_{0}^{\prime} \in F_{0}^{\prime}$ tel que ${ }^{t} u_{0}\left(y_{0}^{\prime}\right) \in$ $\operatorname{Im}^{t} \rho$, on $a: y_{0}^{\prime} \in \operatorname{Im}^{t} \sigma$. 


\section{§4. Homomorphisme Continu du Faisceau $\mathcal{O}$ dans Lui-même}

Proposition 1. Soit $P=p\left(z, \partial_{z}\right)=\sum_{\alpha} a_{\alpha}(z) \partial_{z}^{\alpha}$ un homomorphisme continu de $\mathcal{O}$ dans $\mathcal{O}$. Pour tout $\alpha \in N^{n}$, l'opérateur $p^{(\alpha)}\left(z, \partial_{z}\right)$, ou l'on écrit aussi $P^{(\alpha)}$, est un homomorphisme continu, bien-défini, de $\mathcal{O}$ dans $\mathcal{O}$ au symbole $p^{(\alpha)}(z, \zeta)$ qui est par définition $\partial_{\zeta}^{\alpha} p(z, \zeta)$.

Démonstration. En effet, on a:

$$
\begin{aligned}
& \widetilde{p^{(\alpha)}}(z, \zeta)=\sum_{\beta \geqq \alpha} a_{\beta}(z) \zeta^{\beta-\alpha}\left(\begin{array}{c}
\beta \\
\alpha
\end{array}\right) \alpha !(\beta-\alpha) !, \\
& \tilde{p}^{(\alpha)}(z, \zeta)=\sum_{\beta \geqq \alpha} a_{\beta}(z) \zeta^{\beta-\alpha}\left(\begin{array}{c}
\beta \\
\alpha
\end{array}\right) \alpha ! \beta ! .
\end{aligned}
$$

Comme le symbole $\tilde{p}(z, \zeta)$ est holomorphe dans $\mathbf{C}^{n} \times \mathbf{C}^{n}$, d'où la conclusion.

Proposition 2 (Formule de Leibniz). Soit $p\left(z, \partial_{z}\right)=\sum_{\alpha} a_{\alpha}(z) \partial_{z}^{\alpha}$ un homomorphisme continu de $\mathcal{O}$ dans $\mathcal{O}$. Soient $f$ et $g$ deux fonctions holomorphes dans $\mathbf{C}^{n}$ telles que l'une d'elles soit un polynôme de degré au plus d. Alors on a la formule suivante:

$$
p\left(z, \partial_{z}\right)[f \cdot g]=\sum_{\alpha} \frac{1}{\alpha !} p^{(\alpha)}\left(z, \partial_{z}\right) f \cdot \partial_{z}^{\alpha} g .
$$

Démonstration. Soit $g$ (resp. $f$ ) un polynôme de degré au plus $d$. D'après l'inégalité de Cauchy, il existe $C_{d}$ nombre positif qui ne depend que de $d$ tel que pour tout $z \in \mathbf{C}^{n}$, on ait:

$$
\begin{aligned}
& \sum_{\alpha \leqq \beta}\left(\begin{array}{c}
\beta \\
\alpha
\end{array}\right)\left|\partial_{z}^{\beta-\alpha} f(z)\right| \cdot\left|\partial_{z}^{\alpha} g(z)\right| \\
& \quad \leqq \sum_{|\alpha| \leqq d(\text { resp. }|\beta-\alpha| \leqq d)}\left(\begin{array}{c}
\beta \\
\alpha
\end{array}\right)\|f\|_{\Delta_{1}(z)}\|g\|_{\Delta_{1}(z)}(\beta-\alpha) ! \alpha ! \\
& \quad=C_{d}\|f\|_{\Delta_{1}(z)}\|g\|_{\Delta_{1}(z)} \beta ! .
\end{aligned}
$$

On a donc:

$$
\begin{array}{r}
\sum_{\alpha \leqq \beta, \beta}\left|a_{\beta}(z)\right|\left(\begin{array}{c}
\beta \\
\alpha
\end{array}\right)\left|\partial_{z}^{\beta-\alpha} f(z)\right| \cdot\left|\partial_{z}^{\alpha} g(z)\right| \\
\leqq C_{d}\|f\|_{\Delta_{1}(z)}\|g\|_{\Delta_{1}(z)} \sum_{\beta}\left|a_{\beta}(z)\right| \beta ! .
\end{array}
$$

Comme la série $\sum_{\beta}\left|a_{\beta}(z)\right| \beta$ ! est convergente, on $\mathrm{a}$ :

$$
\begin{array}{r}
p\left(z, \partial_{z}\right)[f \cdot g]=\sum_{\beta} a_{\beta} \partial_{z}^{\beta}[f \cdot g] \\
=\sum_{\beta} a_{\beta}\left(\sum_{\alpha \leqq \beta}\left(\begin{array}{c}
\beta \\
\alpha
\end{array}\right) \partial_{z}^{\beta-\alpha} f \cdot \partial_{z}^{\alpha} g\right)
\end{array}
$$




$$
\begin{aligned}
& =\sum_{\alpha} \partial_{z}^{\alpha} g\left(\sum_{\alpha \leqq \beta} a_{\beta}\left(\begin{array}{c}
\beta \\
\alpha
\end{array}\right) \partial_{z}^{\beta-\alpha} f\right) \\
& =\sum_{\alpha} \partial_{z}^{\alpha} g \frac{1}{\alpha !} p^{(\alpha)}\left(z, \partial_{z}\right) f
\end{aligned}
$$

Proposition 3. Soit $p\left(z, \partial_{z}\right)=\sum_{\alpha} a_{\alpha}(z) \partial_{z}^{\alpha}$ un homomorphisme continu de $\mathcal{O}$ dans $\mathcal{O}$. Pour tout $\eta \in \mathbf{C}^{n}$, l'opérateur $p\left(z, \partial_{z}+\eta\right)$ est un homomorphisme continu, bien-défini, de $\mathcal{O}$ dans $\mathcal{O}$ au symbole $p(z, \zeta+\eta)$.

Démonstration. Pour touts $z \in \mathbb{C}^{n}$ et $\zeta \in \mathbb{C}^{n}$, on a:

$$
\begin{aligned}
& p(z, \zeta+\eta)=\sum_{\alpha} \frac{1}{\alpha !} p^{(\alpha)}(z, \eta) \zeta^{\alpha}, \\
& p^{(\alpha)}(z, \eta)=\sum_{\alpha \leqq \beta} a_{\beta}(z) \eta^{\beta-\alpha}\left(\begin{array}{c}
\beta \\
\alpha
\end{array}\right) \alpha ! 。
\end{aligned}
$$

Pour tout compact $K$ de $\mathbf{C}^{n}$, on a donc:

$$
\begin{aligned}
& \sum_{\alpha}\left\|p^{(\alpha)}(\cdot, \eta)\right\|_{K} \cdot\left|\zeta^{\alpha}\right| \\
& \quad \leqq \sum_{\alpha}\left(\sum_{\alpha \leqq \beta}\left\|a_{\beta}\right\|_{K}\left|\eta^{\beta-\alpha}\right|\left(\begin{array}{c}
\beta \\
\alpha
\end{array}\right) \alpha !\left|\zeta^{\alpha}\right|\right) .
\end{aligned}
$$

D'après la démonstration du théorème 1 de [5], la série

$$
\sum_{\beta}\left\|a_{\beta}\right\|_{K}(|\zeta|+|\eta|)^{|\beta|} \beta !
$$

est convergente. Par suite, on $\mathrm{a}$ :

$$
\begin{aligned}
\sum_{\alpha} & \left(\sum_{\alpha \leqq \beta} a_{\beta}(z) \eta^{\beta-\alpha}\left(\begin{array}{c}
\beta \\
\alpha
\end{array}\right) \beta ! \zeta^{\alpha}\right) \\
& =\sum_{\beta} a_{\beta}(z)\left(\sum_{\alpha \leqq \beta}\left(\begin{array}{c}
\beta \\
\alpha
\end{array}\right) \zeta^{\alpha} \eta^{\beta-\alpha} \beta !\right) \\
& =\sum_{\beta} a_{\beta}(z)(\zeta+\eta)^{\beta} \beta ! \\
& =\tilde{p}(z, \zeta+\eta)
\end{aligned}
$$

et les séries sont normalement convergentes. La série

$$
\sum_{\alpha} \frac{1}{\alpha !} p^{(\alpha)}(z, \eta) \zeta^{\alpha} \alpha !=\sum_{\alpha} p^{(\alpha)}(z, \eta) \zeta^{\alpha}
$$

est donc normalement convergente, d'où la conclusion.

\section{§5. La Fonction Entière $\boldsymbol{p}(\zeta)$}

Lemme 1. Soit $p(\zeta)=\sum_{\alpha} a_{\alpha} \zeta^{\alpha}$ une fonction entière sur $\mathbb{C}^{n}$. Pour que $\tilde{p}(\zeta)=\sum_{\alpha} a_{\alpha} \zeta^{\alpha} \alpha$ ! soit une fonction entière, il faut et il suffit que pour tout nombre 
positif non nul $\varepsilon$, il existe $C_{\varepsilon}$ nombre positif non nul tel que pour tout $\zeta \in \mathbb{C}^{n}$, on ait:

$$
|p(\zeta)| \leqq C_{\varepsilon} \exp (\varepsilon|\zeta|)
$$

Démonstration. Soit $\tilde{p}(\zeta)$ une fonction entière sur $\mathbf{C}^{n}$. D'après l'inégalité de Cauchy, pour tout nombre positif non nul $\varepsilon$ et tout $\alpha \in N^{n}$, on a:

$$
\left|a_{\alpha} \cdot \alpha !\right|=\left|\frac{1}{\alpha !} \tilde{p}^{(\alpha)}(0)\right| \leqq\|\tilde{p}\|_{\Delta_{n} / \varepsilon(0)}(\varepsilon / n)^{|\alpha|} .
$$

On a donc:

$$
\begin{aligned}
|p(\zeta)| & =\left|\sum_{\alpha} a_{\alpha} \zeta^{\alpha}\right| \\
& \leqq\|\tilde{p}\|_{\Delta_{n / \varepsilon}(0)} \sum_{\alpha}\left(\frac{\varepsilon}{n}|\zeta|\right)^{|\alpha|} / \alpha ! \\
& =\|\tilde{p}\|_{\Delta_{n / \varepsilon}(0)} e^{\varepsilon|\zeta|} .
\end{aligned}
$$

Réciproquement, soit l'inégalité (3) vérifiée pour tout nombre positit non nul $\varepsilon$. D'après l'inégalité de Cauchy, pour tout nombre positif non nul $R$ et tout $\alpha \in N^{n}$, on a:

$$
\left|a_{\alpha} \cdot \alpha !\right|=\left|\partial_{\zeta}^{\alpha} p(0)\right| \leqq\|p\|_{\Delta_{R}(0)} R^{-|\alpha|} \alpha ! .
$$

Par l'hypothèse, il existe $C_{\varepsilon}$ nombre positif non nul indépendant de $R$ et $\alpha$ tel que l'on ait:

$$
\|p\|_{\Delta_{R}(0)} \leqq C_{\varepsilon} e^{\varepsilon \sqrt{n} R}
$$

En prenant

$$
R=|\alpha| / \varepsilon \sqrt{n},
$$

on a:

$$
\begin{aligned}
\left|a_{\alpha} \cdot \alpha !\right| & \leqq C_{\varepsilon} e^{|\alpha|}|\alpha|^{-|\alpha|}(\varepsilon \sqrt{n})^{|\alpha| \alpha !} \\
& \leqq C_{\varepsilon} e^{|\alpha| \alpha_{1}^{-\alpha_{1}} \alpha_{2}^{-\alpha_{2}} \cdots \alpha_{n}^{-\alpha_{n}}(\varepsilon \sqrt{n})^{|\alpha|} \alpha ! .}
\end{aligned}
$$

D'après la formule de Stirling, pour tout $\zeta \in \mathbf{C}^{n}$ tel que $|\zeta|<(\varepsilon \sqrt{n})^{-1}$, on a :

$$
\begin{aligned}
& \sup _{\alpha}\left|a_{\alpha} \alpha ! \zeta^{\alpha}\right| \\
& \quad \leqq C_{\varepsilon} \sup _{\alpha} \frac{e^{|\alpha|} \alpha !}{\alpha_{1}^{\alpha_{1} \alpha_{2}^{\alpha} \cdots \alpha_{n}^{\alpha_{n}}}(|\zeta| \varepsilon \sqrt{n})^{|\alpha|}<+\infty .}
\end{aligned}
$$

Comme le nombre positif non nul $\varepsilon$ est arbitraire, d'après le lemme d'Abel, la fonction $\tilde{p}(\zeta)=\sum_{\alpha} a_{\alpha} \alpha ! \zeta^{\alpha}$ est holomorphe dans $\mathbf{C}^{n}$.

Une fonction entière qui vérifie l'inégalité (3) pour tout nombre positif 
non nul $\varepsilon$ est dite de type infra-exponentiel. Donc pour que la fonction $p(\zeta)=$ $\sum_{\alpha} a_{\alpha} \zeta^{\alpha}$ représente un homomorphisme continu de $\mathcal{O}$ dans $\mathcal{O}$ comme le symbole d'un opérateur différentiel à coefficients constants, il faut et il suffit que $p(\zeta)$ soit de type infra-exponentiel.

\section{§6. L'Espace $\boldsymbol{P}_{n}$ des Polynômes}

Pour tout entier positif $d$, on désigne par $P_{n}^{d}$ l'espace des polynômes de degré au plus $d$ à $n$ indéterminées et à coefficients complexes. Comme $P_{n}^{d}$ est un espace vectoriel de dimension finie, il munit canoniquement de la structure d'un espace de Banach. Soient $j_{d}$ l'injection naturelle de $P_{n}^{d}$ dans $P_{n}^{d+1}$ et $\pi_{d}$ la projection canonique de $P_{n}^{d+1}$ sur $P_{n}^{d}$. Soient $P_{n}$ l'espace $(D F S)$ de la limite inductive de la suite $\left(P_{n}^{d}, j_{d}\right)_{d \in N}$ et $Q_{n}$ l'espace $(F S)$ de la limite projective de la suite $\left(P_{n}^{d}, \pi_{d}\right)_{d \in N}$. L'espace localement convexe $P_{n}$ s'identifie avec l'espace des polynômes à $n$ indéterminées ou à $n$ variables complexes $z=\left(z_{1}, z_{2}, \ldots, z_{n}\right)$ et à coefficients complexes et l'espace localement convexe $Q_{n}$ s'identifie avec l'espace des séries formelles à $n$ indéterminées et à coefficients complexes. Les espaces $P_{n}$ et $Q_{n}$ sont en dualité relativement à la forme bilinéaire suivante: pour touts $f=\sum_{\alpha} \frac{1}{\alpha !} \partial^{\alpha} f(0) X^{\alpha} \in P_{n}$ et $u=\sum_{\alpha} \frac{1}{\alpha !} \partial^{\alpha} u(0) X^{\alpha} \in Q_{n}$,

$$
\langle f, u\rangle=\sum_{\alpha} \frac{1}{\alpha !} \partial^{\alpha} f(0) \partial^{\alpha} u(0) .
$$

En outre, les espaces $P_{n}$ et $Q_{n}$ sont même algébriquement en dualité relativement à la forme (4).

Soit $T=\sum_{\alpha} b_{\alpha} \partial^{\alpha}$ un opérateur différentiel formel à coefficients constants sur $P_{n}$, c'est-à-dire que pour tout $f \in P_{n}$, on pose:

$$
T f(X)=\sum_{\alpha} b_{\alpha} \partial^{\alpha} f(X) .
$$

Calculons la transposee ${ }^{t} T: Q_{n} \rightarrow Q_{n}$ de l'opérateur $T: P_{n} \rightarrow P_{n}$ : pour touts $u \in Q_{n}$ et $f \in P_{n}$, on a :

$$
\begin{aligned}
& \left\langle{ }^{t} T u, f\right\rangle=\langle u, T f\rangle \\
& =\sum_{\alpha} \frac{1}{\alpha !} \partial^{\alpha} u(0) \partial^{\alpha}(T f)(0) \\
& =\sum_{\alpha} \frac{1}{\alpha !} \partial^{\alpha} u(0) \sum_{\beta} b_{\beta} \partial^{\beta+\alpha} f(0) \\
& =\sum_{\alpha} \frac{1}{\alpha !} \partial^{\alpha} u(0) \sum_{\alpha \leqq \gamma} b_{\gamma-\alpha} \partial^{\gamma} f(0)
\end{aligned}
$$




$$
\begin{aligned}
& =\sum_{\gamma}\left(\sum_{\alpha \leqq \gamma} b_{\gamma-\alpha} \frac{1}{\alpha !} \partial^{\alpha} u(0)\right) \partial^{\gamma} f(0) \\
& =\langle T(X) \cdot u(X), f(X)\rangle .
\end{aligned}
$$

On a donc:

$$
{ }^{t} T u(X)=T(X) \cdot u(X)
$$

Proposition 4. Soit $T=\sum_{\alpha} b_{\alpha} \partial_{z}^{\alpha}$ un opérateur différentiel formel non nul à coefficients constants sur $P_{n}{ }^{\alpha}{ }^{\alpha}$ Alors on $a: T P_{n}=P_{n}$.

Démonstration. Comme $Q_{n}$ est un anneau d'intégrité, d'après la formule (6), la transposée ${ }^{t} T: Q_{n} \rightarrow Q_{n}$ est injective. Puisque ${ }^{t} T: Q_{n} \rightarrow Q_{n}$ est la transposée algébrique de l'opérateur $T: P_{n} \rightarrow P_{n}$, l'opérateur $T: P_{n} \rightarrow P_{n}$ est surjectif.

\section{§7. L'Espace EP des Exponentielle-Polynômes}

Pour tout $\eta \in \mathbf{C}^{n}$, on désigne par $e_{\eta} P_{n}$ l'espace des fonctions de la forme

$$
e^{\langle\eta, \cdot\rangle} f(\cdot)
$$

avec $f \in P_{n}$ muni de la topologie induite par la topologie de $P_{n}$. Soit $E P$ la somme directe topologique de la famille $\left(e_{\eta} P_{n}\right)_{\eta \in \mathbf{C}^{n}}$ des espaces localement convexes $e_{\eta} P_{n}$. Tout élément de EP est dit un exponentielle-polynôme.

Lemme 2. Le dual $(E P)^{\prime}$ de EP est canoniquement isomorphe à l'espace produit $\prod_{\eta \in \mathbf{C}^{n}} Q_{n}$ relativement à la forme suivante: pour touts $f=\sum_{\eta} e^{\langle\eta, \cdot\rangle} f_{\eta} \in$ EP avec $f_{\eta} \in P_{n}$ et $u=\left(u_{\eta}\right)_{\eta \in \mathbf{C}^{n}} \in \prod_{\eta \in \mathbf{C}^{n}} Q_{n}$,

$$
\langle f, u\rangle=\sum_{\eta}\left\langle f_{\eta}, u_{\eta}\right\rangle \text {. }
$$

Démonstration. On a canoniquement les isomorphismes suivants:

$$
(E P)^{\prime}=\left(\oplus_{\eta} e_{\eta} P_{n}\right)^{\prime} \simeq \prod_{\eta}\left(e_{\eta} P_{n}\right)^{\prime} \simeq \prod_{\eta} P_{n}^{\prime}=\prod_{\eta} Q_{n},
$$

d'où la conclusion.

Proposition 5. Soit $p\left(\partial_{z}\right)$ un opérateur différentiel non nul à coefficients constants représenté par un homomorphisme continu $P$ de $\mathcal{O}$ dans $\mathcal{O}$. Alors on $a: p\left(\partial_{z}\right) E P=E P$.

Démonstration. Pour tout $f \in E P$, il existe $\left(f_{j}\right)_{1 \leqq j \leqq l}$ suite finie de $P_{n}$ tel que l'on ait: 


$$
f=\sum_{j=1}^{l} e^{\left\langle\eta_{j}, \cdot\right\rangle} f_{j}
$$

D'après la proposition 4 , il existe $g_{j} \in P_{n}$ tel que l'on ait:

$$
p\left(\partial_{z}+\eta_{j}\right) g_{j}=f_{j}
$$

pour tout $j$. On pose:

$$
g=\sum_{j=1}^{l} e^{\left\langle\eta_{j},{ }^{\circ}\right\rangle} g_{j} \in E P .
$$

D'après la proposition 2 et la proposition 3 , on $\mathrm{a}$ :

$$
\begin{aligned}
p\left(\partial_{z}\right) g & =\sum_{j} p\left(\partial_{z}\right)\left(e^{\left\langle\eta_{j}, \cdot\right\rangle} g_{j}\right) \\
& =\sum_{j} e^{\left\langle\eta_{j}, \cdot\right\rangle} \sum_{\alpha} \frac{1}{\alpha !} p^{(\alpha)}\left(\eta_{j}\right) \partial_{z}^{\alpha} g_{j} \\
& =\sum_{j} e^{\left\langle\eta_{j}, \cdot\right\rangle} p\left(\partial_{z}+\eta_{j}\right) g_{j} \\
& =\sum_{j} e^{\left\langle\eta_{j}, \cdot\right\rangle} f_{j} \\
& =f
\end{aligned}
$$

\section{§8. Existence et Approximation dans l'Espace des Fonctions Entières}

Dans cette section, on désignera par $p\left(\partial_{z}\right)$ un opérateur différentiel non nul à coefficients constants représenté par un homomorphisme continu $P$ de $\mathcal{O}$ dans $\mathcal{O}$. D'après la démonstration de la proposition 5 , on a la formule suivante: pour tout $f=\sum_{\eta} e^{\langle\eta, \cdot\rangle} f_{\eta} \in E P$ avec $f_{\eta} \in P_{n}$,

$$
p\left(\partial_{z}\right) f=\sum_{\eta} e^{\langle\eta, \cdot\rangle} p\left(\partial_{z}+\eta\right) f_{\eta} .
$$

Calculons la transposée ${ }^{t} p\left(\partial_{z}\right):(E P)^{\prime} \rightarrow(E P)^{\prime}$ de $p\left(\partial_{z}\right): E P \rightarrow E P:$ pour touts $f=\sum_{\eta} e^{\langle\eta, \cdot\rangle} f_{\eta} \in E P$ avec $f_{\eta} \in P_{n}$ et $u=\left(u_{\eta}\right)_{\eta \in \mathbb{C}^{n}} \in \prod_{\eta \in \mathbb{C}^{n}} Q_{n}=(E P)^{\prime}$, on a:

$$
\begin{aligned}
\left\langle{ }^{t} p\left(\partial_{z}\right) u, f\right\rangle & =\left\langle u, p\left(\partial_{z}\right) f\right\rangle \\
& =\left\langle u, \sum_{\eta} e^{\langle\eta, \cdot\rangle} p\left(\partial_{z}+\eta\right) f_{\eta}\right\rangle \\
& =\sum_{\eta}\left\langle u_{\eta}, p\left(\partial_{z}+\eta\right) f_{\eta}\right\rangle \\
& =\sum_{\eta}\left\langle p(\zeta+\eta) u_{\eta}(\zeta), f_{\eta}(\zeta)\right\rangle_{\zeta} .
\end{aligned}
$$

En posant

$$
\left(\tau_{-\eta} h\right)(\zeta)=h(\zeta+\eta)
$$


d'après le lemme 2 , on a:

$$
{ }^{t} p\left(\partial_{z}\right) u=\left(\left(\tau_{-\eta} p\right) u_{\eta}\right)_{\eta \in \mathbf{C}} .
$$

Maintenant, considérons le diagramme commutatif suivant:

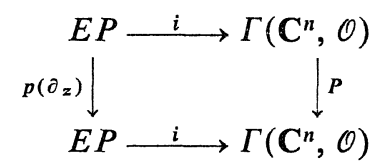

où $i$ est l'injection naturelle continue.

Proposition 6. Dans le diagramme (10), la surjection $p\left(\partial_{z}\right): E P \rightarrow E P$ est continue.

Démonstration. Il suffit de montrer que pour tout $\eta \in \mathbb{C}^{n}$ et tout entier positif $d$, l'opérateur $p\left(\partial_{z}\right): e_{\eta} P_{n}^{d} \rightarrow E P$ est continu. Soit $\left(f_{v}\right)_{v \geqq 1}$ une suite convergeant dans $P_{n}^{d}$ à 0 . Alors $\left(f_{v}\right)_{v \geqq 1}$ converge dans l'espace $\Gamma\left(\mathbf{C}^{n}, \mathcal{O}\right)$ à 0 . D'après l'égalité (8), on a:

$$
p\left(\partial_{z}\right)\left(e^{\langle\eta, \cdot\rangle} f_{v}\right)=e^{\langle\eta, \cdot\rangle} p\left(\partial_{z}+\eta\right) f_{v} \in e_{\eta} P_{n}^{d} .
$$

Puisque l'opérateur $p\left(\partial_{z}+\eta\right): \Gamma\left(\mathbf{C}^{n}, \mathcal{O}\right) \rightarrow \Gamma\left(\mathbf{C}^{n}, \mathcal{O}\right)$ est continu, la suite $\left(p\left(\partial_{z}+\eta\right) f_{v}\right)_{v \geqq 1}$ converge dans $\Gamma\left(\mathbb{C}^{n}, \mathcal{O}\right)$ à 0 . Comme l'injection naturelle: $P_{n}^{d} \rightarrow \Gamma\left(\mathbf{C}^{n}, \mathcal{O}\right)$ est monomorphisme, la suite $\left(p\left(\partial_{z}+\eta\right) f_{v}\right)_{v \geqq 1}$ converge dans $P_{n}^{d}$ à 0 , donc dans $P_{n}$ à 0 .

Corollaire. Dans la situation précédente, la surjection $p\left(\partial_{z}\right): P_{n} \rightarrow P_{n}$ est un epimorphisme.

Démonstration. D'après la démonstration de la proposition précédente, la surjection $p\left(\partial_{z}\right): P_{n} \rightarrow P_{n}$ est continue. D'après le corollaire de la proposition 10 de la section 4 du chapitre II de Bourbaki [2], d'où la conclusion.

Lemme 3. Dans la situation précédente, l'idéal $p(X) \cdot Q_{n}$ est fermé dans l'espace $Q_{n}$.

Démonstration. La surjection $p\left(\partial_{z}\right): P_{n} \rightarrow P_{n}$ est un epimorphisme. En posant

$$
N=\operatorname{Ker} p\left(\partial_{z}\right)
$$

l'application canonique

$$
\bar{p}: P_{n} / N \longrightarrow P_{n}
$$


induite par $p\left(\partial_{z}\right): P_{n} \rightarrow P_{n}$ est donc un isomorphisme. Par suite, on a la bijection

$$
{ }^{t} \bar{p}: Q_{n} \longrightarrow N^{0} \subset Q_{n}
$$

et ${ }^{t} \bar{p} Q_{n}=N^{0}$ est fermé dans $Q_{n}$. Puisque l'on a:

$$
p(X) \cdot Q_{n}={ }^{t} \bar{p} Q_{n},
$$

l'idéal $p(X) \cdot Q_{n}$ est fermé dans $Q_{n}$.

Lemme 4. Dans la situation précédente, l'ensemble ${ }^{t} p\left(\partial_{z}\right)(E P)^{\prime}$ est fermé dans $(E P)^{\prime}$, où $(E P)^{\prime}$ munit de la topologie induite par l'espace $\prod_{\eta \in \mathbf{C}^{n}} Q_{n}$. (9), on a:

Démonstration. Pour tout $u=\left(u_{\eta}\right)_{\eta \in \mathbf{C}^{n}} \in \prod_{\eta \in \mathbf{C}^{n}} Q_{n}=(E P)^{\prime}$, d'après l'égalité

$$
{ }^{t} p\left(\partial_{z}\right) u=\left(\left(\tau_{-\eta} p\right) u_{\eta}\right)_{\eta \in \mathbf{C}^{n}}
$$

On a donc:

$$
{ }^{t} p\left(\partial_{z}\right)(E P)^{\prime}=\prod_{\eta \in \mathbf{C}^{n}} \tau_{-\eta} p(X) \cdot Q_{n} .
$$

D'après le lemme 3 , pour tout $\eta \in \mathbb{C}^{n}$, l'ensemble $\tau_{-\eta} p(x) \cdot Q_{n}$ est fermé dans $Q_{n}$. D'où la conclusion.

Lemme 5. Dans la situation précédente, on $a$ :

$$
{ }^{t} p\left(\partial_{z}\right)(E P)^{\prime}=\left(\operatorname{Ker} p\left(\partial_{z}\right)\right)^{0} .
$$

Démonstration. On a algébriquement les isomorphismes canoniques suivants:

$$
\left(\prod_{\eta \in \mathbf{C}^{n}} Q_{n}\right)^{\prime} \simeq \underset{\eta \in \mathbf{C}^{n}}{\oplus} Q_{n}^{\prime}=\underset{\eta \in \mathbf{C}^{n}}{\oplus} P_{n} \simeq \bigoplus_{\eta \in \mathbf{C}^{n}} e_{\eta} P_{n}=E P .
$$

Par suite, la topologie de $(E P)^{\prime}=\prod_{\eta \in \mathbf{C}^{n}} Q_{n}$ est compatible avec la dualité entre $(E P)^{\prime}$ et $E P$. On a donc:

$$
\overline{\operatorname{Im}^{t} p\left(\partial_{z}\right)}=\left(\operatorname{Ker} p\left(\partial_{z}\right)\right)^{0}
$$

dans $\prod_{\eta \in \mathbf{C}^{n}} Q_{n}=(E P)^{\prime}$. D'après le lemme 4, d'où la conclusion.

Dans le diagramme (10), la surjection $p\left(\partial_{z}\right): E P \rightarrow E P$ est essentiellement univalente. On va démontrer que l'opérateur $P: \Gamma\left(\mathbb{C}^{n}, \mathcal{O}\right) \rightarrow \Gamma\left(\mathbb{C}^{n}, \mathcal{O}\right)$ est l'extension canonique de $p\left(\partial_{z}\right): E P \rightarrow E P:$ Comme l'opérateur $P: \Gamma\left(\mathbb{C}^{n}, \mathcal{O}\right) \rightarrow$ $\Gamma\left(\mathbf{C}^{n}, \mathcal{O}\right)$ est continu, son graphe $G$ est fermé dans l'espace produit $\Gamma\left(\mathbb{C}^{n}, \mathcal{O}\right)$ $\times \Gamma\left(\mathbf{C}^{n}, \mathcal{O}\right)$. Soit $G_{0}$ le graphe de $p\left(\partial_{z}\right): E P \rightarrow E P$ dans l'espace $E P \times E P \subset$ $\Gamma\left(\mathbf{C}^{n}, \mathcal{O}\right) \times \Gamma\left(\mathbf{C}^{n}, \mathcal{O}\right)$. Comme l'espace $E P$ est dense dans l'espace $\Gamma\left(\mathbb{C}^{n}, \mathcal{O}\right)$, 
pour tout couple $(f, P f) \in G$, il existe $\left(f_{v}\right)_{v \geqq 1}$ suite de $E P$ qui converge dans $\Gamma\left(\mathbf{C}^{n}, \mathcal{O}\right)$ à $f$. Puisque l'opérateur $P: \Gamma\left(\mathbf{C}^{n}, \mathcal{O}\right) \rightarrow \Gamma\left(\mathbf{C}^{n}, \mathcal{O}\right)$ est continu, la suite $\left(\left(f_{v}, P f_{v}\right)\right)_{v \geqq 1}$ de $G_{0}$ converge dans $\Gamma\left(\mathbf{C}^{n}, \mathcal{O}\right) \times \Gamma\left(\mathbf{C}^{n}, \mathcal{O}\right)$ à $(f, P f)$. On a donc: $G=\overline{G_{0}}$.

Comme l'espace $E P$ est dense dans l'espace $\Gamma\left(\mathbf{C}^{n}, \mathcal{O}\right)$, d'après le critère 2, les énoncés suivants sont équivalents:

(a) L'opérateur $P: \Gamma\left(\mathbf{C}^{n}, \mathcal{O}\right) \rightarrow \Gamma\left(\mathbf{C}^{n}, \mathcal{O}\right)$ est un epimorphisme ayant la propriété d'approximation homogène.

(b) Pour tout $u \in(E P)^{\prime}$ tel que ${ }^{t} p\left(\partial_{z}\right) u \in{ }^{t} i\left(\Gamma\left(\mathbf{C}^{n}, \mathcal{O}\right)^{\prime}\right)$, on a: $u \in{ }^{t} i\left(\Gamma\left(\mathbf{C}^{n}, \mathcal{O}\right)^{\prime}\right)$.

Par la transformation de Fourier-Borel $\mathscr{F}$, l'espace $\Gamma\left(\mathbf{C}^{n}, \mathcal{O}\right)^{\prime}$ s'identifie avec Exp espace des fonctions entières de type exponentiel. Calculons la transposée ${ }^{i} i: \operatorname{Exp} \rightarrow \prod_{\eta \in \mathbf{C}^{n}} Q_{n}$ de $i: E P \rightarrow \Gamma\left(\mathbf{C}^{n}, \mathcal{O}\right):$ pour tout $h \in \operatorname{Exp}$ et tout $f=\sum_{\eta} e^{\langle\eta, \cdot\rangle} f_{\eta}$ $\in E P$ avec $f_{\eta} \in P_{n}$, en prenant une fonctionnelle analytique $T \in \Gamma\left(\mathbf{C}^{n},{ }^{\eta} \mathcal{O}\right)^{\prime}$ telle que l'on ait: $h=\mathscr{F} T$, on a:

$$
\begin{aligned}
\left\langle{ }^{i} i h, f\right\rangle & =\left\langle{ }^{t} i T, f\right\rangle=\langle T, f\rangle \\
& =\sum_{\eta}\left\langle T, e^{\langle\eta, \cdot\rangle} f_{\eta}\right\rangle \\
& =\sum_{\eta} \sum_{\alpha} \partial_{z}^{\alpha}(\mathscr{F} T)(0) \partial_{z}^{\alpha}\left(e^{\langle\eta, \cdot\rangle} f_{\eta}\right)(0) / \alpha ! \\
& =\sum_{\eta} \sum_{\alpha} \partial_{z}^{\alpha} h(0)\left(\sum_{\beta \leqq \alpha}\left(\begin{array}{c}
\alpha \\
\beta
\end{array}\right) \eta^{\beta} \partial_{z}^{\alpha-\beta} f_{\eta}(0)\right) / \alpha ! \\
& =\sum_{\eta} \sum_{\beta}\left(\sum_{\beta \leqq \alpha} \partial_{z}^{\alpha} h(0)\left(\begin{array}{c}
\alpha \\
\beta
\end{array}\right) \partial_{z}^{\alpha-\beta} f_{\eta}(0) / \alpha !\right) \eta^{\beta} \\
& =\sum_{\eta} \sum_{\beta}\left(\sum_{\alpha \geqq 0} \partial_{z}^{\alpha+\beta} h(0)\left(\begin{array}{c}
\alpha+\beta \\
\beta
\end{array}\right) \partial_{z}^{\alpha} f_{\eta}(0) /(\alpha+\beta) !\right) \eta^{\beta} \\
& =\sum_{\eta} \sum_{\beta}\left(\sum_{\alpha} \partial_{z}^{\alpha}\left(\partial_{z}^{\beta} h\right)(0) \partial_{z}^{\alpha} f(0) / \alpha !\right) \eta^{\beta} / \beta ! \\
& =\sum_{\eta} \sum_{\beta}\left\langle\partial_{z}^{\beta} h, f_{\eta}\right\rangle \eta^{\beta} / \beta ! \\
& =\sum_{\eta}\left\langle\tau_{-\eta} h, f_{\eta}\right\rangle .
\end{aligned}
$$

On a donc:

$$
{ }^{t} i h=\left(\tau_{-\eta} h\right)_{\eta \in \mathbf{C}^{n}} .
$$

Par suite, d'après la formule (9), l'énoncé (b) est équivalent à l'énoncé suivant:

(b)' Pour touts $u=\left(u_{\eta}\right)_{\eta \in \mathbf{C}^{n}} \in \prod_{\eta \in \mathbf{C}^{n}} Q_{n}$ et $h \in \operatorname{Exp}$ tel que l'on ait:

$$
\left(\tau_{-\eta} p\right) \cdot u_{\eta}=\tau_{-\eta} h
$$

pour tout $\eta \in \mathbf{C}^{n}$, il existe $g \in \operatorname{Exp}$ tel que l'on ait: 


$$
u_{\eta}=\tau_{-\eta} g
$$

pour tout $\eta \in \mathbb{C}^{n}$.

Théorème 1. Soit $p\left(\partial_{z}\right)=\sum_{\alpha} a_{\alpha} \partial_{z}^{\alpha}$ un opérateur différentiel non nul ̀̀ coefficients constants sur $\mathbb{C}^{n}$ représenté par un homomorphisme continu $P$ de $\mathcal{O}$ dans $\mathcal{O}$. Alors on a les énoncés suivants (i) et (ii):

(i) Pour toute fonction entière $f$ sur $\mathbb{C}^{n}$, il existe $u$ fonction entière sur $\mathrm{C}^{n}$ tel que l'on ait:

$$
p\left(\partial_{z}\right) u=f
$$

dans $\mathbb{C}^{n}$.

(ii) Toute solution u dans $\Gamma\left(\mathbb{C}^{n}, \mathcal{O}\right)$ de l'équation homogène

$$
p\left(\partial_{z}\right) u=0
$$

est approchée dans $\Gamma\left(\mathbb{C}^{n}, \mathcal{O}\right)$ par des exponentielle-polynômes satisfaisant à l'équation (13).

Démonstration. On va démontrer que l'énoncé (b)' est valable. Soient $u=\left(u_{\eta}\right)_{\eta \in \mathbf{C}^{n}}$ un élément de $\prod_{\eta \in \mathbb{C}^{n}} Q_{n}$ et $h$ un élément de $\operatorname{Exp}$ tels que l'on ait:

$$
\left(\tau_{-\eta} p\right) u_{\eta}=\tau_{-\eta} h
$$

pour tout $\eta \in \mathbb{C}^{n}$. D'après le Vorbereitungsatz donné, par exemple, dans Trèves [7], pour tout $\eta \in \mathbb{C}^{n}, u_{\eta}$ est holomorphe dans un voisinage de $0 \in \mathbb{C}^{n}$. On pose:

$$
g_{\eta}(\zeta)=u_{\eta}(\zeta-\eta)
$$

pour touts $\eta \in \mathbb{C}^{n}$ et $\zeta \in \mathbb{C}^{n}$. Pour tout $\eta \in \mathbb{C}^{n}$, il existe $U_{\eta}$ voisinage de $\eta$ tel que l'on ait: $g_{\eta} \in \Gamma\left(U_{\eta}, \mathcal{O}\right)$. Pour tout point $\zeta$ de $U_{\eta}$, on a:

$$
p(\zeta) g_{\eta}(\zeta)=p(\zeta) u_{\eta}(\zeta-\eta)=h(\zeta) \text {. }
$$

Puisque la fonction entière $p(\zeta)$ n'est pas nulle, pour touts points $\eta$ et $\eta^{\prime}$ de $\mathbb{C}^{n}$, on a donc:

$$
g_{\eta}=g_{\eta^{\prime}}
$$

dans $U_{\eta} \cap U_{\eta^{\prime}}$. Il existe donc $g$ fonction entière tel que pour tout $\eta \in \mathbb{C}^{n}$, on ait:

$$
g_{\mid U_{\eta}}=g_{\eta}=\tau_{\eta} u_{\eta}
$$

dans $U_{\eta^{\circ}}$ On a par suite: 


$$
\tau_{-\eta} g=u_{\eta}
$$

pour tout $\eta \in \mathbf{C}^{n}$ et l'on a:

$$
p(\zeta) g(\zeta)=h(\zeta)
$$

Comme les fonctions entières $p(\zeta)$ et $h(\zeta)$ sont de type exponentiel, d'après le théorème 1 du chapitre II de Malgrange [6], la fonction $g$ l'est aussi, d'où la conclusion.

\section{§9. Existence et Approximation dans un Ouvert de $\mathbf{C}^{n}$}

Dans cette section, on désigne par $p\left(\partial_{z}\right)$ un opérateur différentiel non nul a coefficients constants représenté par un homomorphisme continu $P$ de $\mathcal{O}$ dans $\mathcal{O}$ et par $\Omega$ un ouvert de type de Runge dans $\mathbf{C}^{n}$, c'est-à-dire que $\Omega$ est un ouvert de $\mathbf{C}^{n}$ et la restriction

$$
r_{\Omega}: \Gamma\left(\mathbf{C}^{n}, \mathcal{O}\right) \longrightarrow \Gamma(\Omega, \mathcal{O})
$$

a l'image dense dans $\Gamma(\Omega, \mathcal{O})$. Considérons le diagramme commutatif suivant:

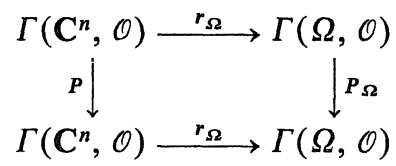

D'après le théorème 1 , l'opérateur

$$
P: \Gamma\left(\mathbf{C}^{n}, \mathcal{O}\right) \longrightarrow \Gamma\left(\mathbf{C}^{n}, \mathcal{O}\right)
$$

est surjectif et essentiellement univalent. On va démontrer que l'opérateur

$$
P_{\Omega}: \Gamma(\Omega, \mathcal{O}) \longrightarrow \Gamma(\Omega, \mathcal{O})
$$

est l'extension canonique de $P$ : Comme l'opérateur $P_{\Omega}$ est continu, son graphe $G_{\Omega}$ est fermé dans l'espace produit $\Gamma(\Omega, \mathcal{O}) \times \Gamma(\Omega, \mathcal{O})$. Soit $G_{1}$ le graphe de $P$ dans l'espace $\Gamma\left(\mathbf{C}^{n}, \mathcal{O}\right) \times \Gamma\left(\mathbf{C}^{n}, \mathcal{O}\right)$ et l'on pose:

$$
G=\left(r_{\Omega} \times r_{\Omega}\right)\left(G_{1}\right) .
$$

Puisque l'espace $\Gamma\left(\mathbb{C}^{n}, \mathcal{O}\right)$ est dense dans l'espace $\Gamma(\Omega, \mathcal{O})$, pour tout couple $\left(f, P_{\Omega} f\right) \in G_{\Omega}$, il existe $\left(f_{v}\right)_{v \geqq 1}$ suite de $\Gamma\left(\mathbf{C}^{n}, \mathcal{O}\right)$ qui converge dans $\Gamma(\Omega, \mathcal{O})$ à $f$. Comme l'opérateur $P_{\Omega}: \Gamma(\Omega, \mathcal{O}) \rightarrow \Gamma(\Omega, \mathcal{O})$ est continu, la suite $\left(\left(f_{v}, P_{\Omega} f_{v}\right)\right)_{v \geqq 1}$ de $G$ converge dans $\Gamma(\Omega, \mathcal{O}) \times \Gamma(\Omega, \mathcal{O})$ à $\left(f, P_{\Omega} f\right)$. On a donc: $G_{\Omega}=\bar{G}$.

On dira qu'une fonctionnelle analytique $T \in \Gamma\left(\mathbf{C}^{n}, \mathcal{O}\right)^{\prime}$ est appuyée par 
louvert $\Omega$ si $T$ appartient à l'image de la transposée

$$
{ }^{t} r_{\Omega}: \Gamma(\Omega, \mathcal{O})^{\prime} \longrightarrow \Gamma\left(\mathbf{C}^{n}, \mathcal{O}\right)^{\prime}
$$

de la restriction $r_{\Omega}: \Gamma\left(\mathbf{C}^{n}, \mathcal{O}\right) \rightarrow \Gamma(\Omega, \mathcal{O})$.

Définition 4. Soient $p\left(\partial_{z}\right)$ un opérateur différentiel à coefficients constants sur $\mathbf{C}^{n}$ représenté par un homomorphisme continu $P$ de $\mathcal{O}$ dans $\mathcal{O}$ et $\Omega$ un ouvert de $\mathbf{C}^{n}$. On dit que $\Omega$ est pseudo $p\left(\partial_{z}\right)$-convexe si l'énoncé suivant est vérifié: si $T$ est une fonctionnelle analytique sur $\mathbf{C}^{n}$ telle que la fonctionnelle analytique ${ }^{t} P T$ soit appuyée par $\Omega, T$ est appuyée par $\Omega$ où ${ }^{t} P$ désigné la transposée de l'opérateur $P: \Gamma\left(\mathbf{C}^{n}, \mathcal{O}\right) \rightarrow \Gamma\left(\mathbf{C}^{n}, \mathcal{O}\right)$ induite par l'homomorphisme $P: \mathcal{O} \rightarrow \mathcal{O}$.

D'après le critère 3 et le théorème 1 , (ii), on a le théorème suivant:

Théorème 2. Soient $p\left(\partial_{z}\right)=\sum_{\alpha} a_{\alpha} \partial_{z}^{\alpha}$ un opérateur différentiel non nul à coefficients constants sur $\mathbf{C}^{n}$ représenté par un homomorphisme continu $P$ de $\mathcal{O}$ dans $\mathcal{O}$ et $\Omega$ un ouvert de type de Runge dans $\mathbb{C}^{n}$. Alors pour que les énoncés suivants (i) et (ii) soient valables, il faut et il suffit que l'ouvert $\Omega$ soit pseudo $p\left(\partial_{z}\right)$-convexe:

(i) Pour toute fonction holomorphe $f$ sur $\Omega$, il existe $u$ fonction holomorphe sur $\Omega$ tel que l'on ait:

$$
p\left(\partial_{z}\right) u=f
$$

dans $\Omega$.

(ii) Toute solution u dans $\Gamma(\Omega, \mathcal{O})$ de l'équation homogène

$$
p\left(\partial_{z}\right) u=0
$$

est approchée dans $\Gamma(\Omega, \mathcal{O})$ par des exponentielle-polynômes satisfaisant à l'équation (16).

Proposition 7. Soient $p\left(\partial_{z}\right)$ un opérateur différentiel non nul à coefficients constants sur $\mathbf{C}^{n}$ représenté par un homomorphisme continu de $\mathcal{O}$ dans $\mathcal{O}$ et $\Omega$ un ouvert convexe de $\mathbf{C}^{n}$. Alors $\Omega$ est pseudo $p\left(\partial_{z}\right)$-convexe.

Pour démontrer la proposition 7, d'après Avanissian [1], on va démontrer d'abord trois lemmes. Pour toute fonction Lebesgue-mesurable $g$ de $\mathbf{C}$ dans $\mathbf{C}$, tout $z_{0} \in \mathbf{C}$ et tout nombre positif non nul $r$, on pose:

$$
M\left(g ; z_{0}, r\right)=\frac{1}{\pi r^{2}} \int_{\left|\zeta-z_{0}\right|<r} g d \mu_{\zeta}
$$

où $d \mu_{\zeta}$ désigné la mesure de Lebesgue en $\zeta$ dans $\mathbf{C}$. 
Lemme 6. Soient $A$ un nombre positif non nul et $g$ une fonction entière sur $\mathbf{C}$ telle que $g(0) \neq 0$ et que avec un nombre positif $K$ plus grand que 1 , on ait:

$$
|g(\zeta)| \leqq K e^{A|\zeta|}
$$

pour tout $\zeta \in \mathbf{C}$. Alors pour tout nombre positif non nul $\lambda$, tout nombre $A^{\prime}$ tel que $A^{\prime}>A$ et tout $z \in \mathbf{C}$ tel que $|z|>(\log K) /\left(A^{\prime}-A\right)$, on $a$ :

$$
\begin{aligned}
& M(\log |g| ; z, \lambda|z|) \\
& \quad \geqq\left(\frac{1+\lambda}{\lambda}\right)^{2} \log |g(0)|+\left(1-\left(\frac{1+\lambda}{\lambda}\right)^{2}\right) A^{\prime}(1+\lambda)|z| .
\end{aligned}
$$

Démonstration. Supposons que l'on a:

$$
\begin{aligned}
& \lambda>0, A^{\prime}>A, \\
& |z|>\frac{\log K}{A^{\prime}-A} .
\end{aligned}
$$

On pose:

$$
\begin{aligned}
& R=(1+\lambda) \cdot|z|, \\
& v(\zeta)=\log |g(\zeta)|-A^{\prime} R .
\end{aligned}
$$

Pour tout $\zeta \in \mathbf{C}$ tel que $|\zeta| \leqq R$, on a:

$$
\begin{aligned}
v(\zeta) & \leqq A|\zeta|+\log K-A^{\prime} R \\
& \leqq\left(A-A^{\prime}\right) R+\log K \\
& =\left(A-A^{\prime}\right)(1+\lambda)|z|+\log K \\
& <-(1+\lambda) \log K+\log K \leqq 0 .
\end{aligned}
$$

Par suite, on a:

$$
v<0
$$

dans $\{\zeta \in \mathrm{C}|| \zeta \mid \leqq R\}$. Puisque l'on a:

$$
\{\zeta \in \mathbf{C}|| \zeta \mid \leqq R\} \supset\{\zeta \in \mathbf{C}|| \zeta-z|\leqq \lambda| z \mid\},
$$

on a:

$$
\int_{|\zeta| \leqq R} v d \mu_{\zeta} \leqq \int_{|\zeta-z| \leqq \lambda|z|} v d \mu_{\zeta}
$$

c'est-à-dire que l'on a:

$$
R^{2} M(v ; 0, R) \leqq \lambda^{2}|z|^{2} M(v ; z, \lambda|z|) .
$$

Comme la fonction $v$ est sous-harmonique, on a: 


$$
\begin{aligned}
R^{2}\left(\log |g(0)|-A^{\prime} R\right) & =R^{2} v(0) \\
& \leqq R^{2} M(v ; 0, R)
\end{aligned}
$$

On a donc:

$$
\begin{aligned}
& (1+\lambda)^{2}|z|^{2}\left(\log |g(0)|-A^{\prime} R\right) \\
& \quad \leqq \lambda^{2}|z|^{2} M(v ; z, \lambda|z|) \\
& \quad=\lambda^{2}|z|^{2}\left[M(\log |g| ; z, \lambda|z|)-A^{\prime} R\right] .
\end{aligned}
$$

Puisque $|z| \neq 0$, on a donc:

$$
\begin{aligned}
& M(\log |g| ; z, \lambda|z|) \\
& \quad \geqq\left(\frac{1+\lambda}{\lambda}\right)^{2} \log |g(0)|+\left(1-\left(\frac{1+\lambda}{\lambda}\right)^{2}\right) A^{\prime}(1+\lambda)|z| .
\end{aligned}
$$

Lemme 7. Soient $A$ et $B$ deux nombres positifs non nuls et $f$ et $g$ deux fonctions entières sur $\mathbf{C}$ telles que $g(0) \neq 0$, que le quotient $\mathrm{f} / \mathrm{g}$ est une fonction entière et que avec deux nombres positifs $K$ et $L$ plus grands que 1 , on ait:

$$
\begin{aligned}
& |f(\zeta)| \leqq K e^{A|\zeta|+J(\zeta)}, \\
& |g(\zeta)| \leqq L e^{B|\zeta|}
\end{aligned}
$$

pour tout $\zeta \in \mathbb{C}$, où $J$ est une fonction Lebesgue-mesurable de $\mathbf{C}$ dans $\mathbf{R}$. Alors pour tout nombre positif non nul $\lambda$, touts nombres $A^{\prime}$ et $B^{\prime}$ tels que $A^{\prime}>A$, $B^{\prime}>B$ et tout $z \in \mathbf{C}$ tel que

$$
|z|>\sup \left[\frac{\log K}{A^{\prime}-A}, \frac{\log L}{B^{\prime}-B}\right],
$$

on $a$ :

(19) $|f / g(z)|$

$$
\leqq|g(0)|^{-\left(\frac{1+\lambda}{\lambda}\right)^{2}} \exp \left[J_{\lambda}(z)+\left\{A^{\prime}(1+\lambda)+B^{\prime}(1+\lambda)\left(\left(\frac{1+\lambda}{\lambda}\right)^{2}-1\right)\right\}|z|\right],
$$

où l'on se rappelle:

$$
J_{\lambda}(z)=\sup _{|\zeta-z|<\lambda|z|} J(\zeta)
$$

Démonstration. Supposons que l'on a:

$$
\begin{aligned}
& \lambda>0, A^{\prime}>A, B^{\prime}>B, \\
& |z|>\sup \left[\frac{\log K}{A^{\prime}-A}, \frac{\log L}{B^{\prime}-B}\right] .
\end{aligned}
$$

Comme la fonction $\log |f / g|$ est sous-harmonique, on a: 


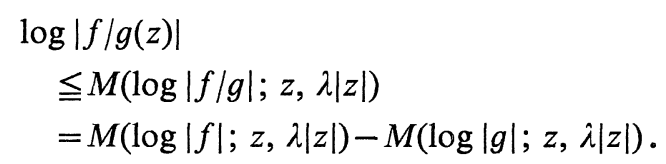

On a:

$$
\begin{aligned}
M & (\log |f| ; z, \lambda|z|)=\frac{1}{\lambda^{2}|z|^{2}} \int_{|\zeta-z|<\lambda|z|} \log |f| d \mu_{\zeta} \\
& \leqq \frac{1}{\lambda^{2}|z|^{2}} \int_{|\zeta-z|<\lambda|z|}(J(\zeta)+A|\zeta|+\log K) d \mu_{\zeta} \\
& \leqq \log K+A(1+\lambda)|z|+J_{\lambda}(z) \\
& =\log K-\left(A^{\prime}-A\right)(1+\lambda)|z|+A^{\prime}(1+\lambda)|z|+J_{\lambda}(z) \\
& <\log K-\log K+A^{\prime}(1+\lambda)|z|+J_{\lambda}(z) \\
& =A^{\prime}(1+\lambda)|z|+J_{\lambda}(z) .
\end{aligned}
$$

D'après l'inégalité (18), on a donc:

$$
\begin{aligned}
& \log |f| g(z) \mid \\
& \quad \leqq J_{\lambda}(z)+A^{\prime}(1+\lambda)|z|-\left(\frac{1+\lambda}{\lambda}\right)^{2} \log |g(0)|+\left(\left(\frac{1+\lambda}{\lambda}\right)^{2}-1\right) B^{\prime}(1+\lambda)|z| .
\end{aligned}
$$

Lemme 8. Soient $A$ et $B$ deux nombres positifs non nuls et $f$ et $g$ deux fonctions entières sur $\mathbf{C}^{n}$ telles que $g(0) \neq 0$, que le quotient $\mathrm{f} / \mathrm{g}$ est une fonction entière et que avec deux nombres positifs $K$ et $L$ plus grands que 1 , on ait:

$$
\begin{aligned}
& |f(\zeta)| \leqq K e^{A|\zeta|+J(\zeta)} \\
& |g(\zeta)| \leqq L e^{B|\zeta|}
\end{aligned}
$$

pour tout $\zeta \in \mathbf{C}^{n}$, où $J$ est une fonction Lebesgue-mesurable de $\mathbf{C}^{n}$ dans $\mathbf{R}$. Alors pour tout nombre positif non nul $\lambda$ et touts nombres $A^{\prime}$ et $B^{\prime}$ tels que $A^{\prime}>A, B^{\prime}>B$, il existe C nombre positif non nul tel que l'on ait:

$$
|f| g(z) \mid \leqq C \exp \left[J_{\lambda}(z)+\left\{A^{\prime}(1+\lambda)+B^{\prime}(1+\lambda)\left(\left(\frac{1+\lambda}{\lambda}\right)^{2}-1\right)\right\}|z|\right]
$$

pour tout $z \in \mathbf{C}^{n}$, où l'on se rappelle:

$$
J_{\lambda}(z)=\sup _{|\zeta-z|<\lambda|z|} J(\zeta) .
$$

Démonstration. Pour tout $z \in \mathbf{C}^{n}$ tel que $|z|=1$, on pose:

$$
\begin{aligned}
& f_{z}: t \longmapsto f\left(t z_{1}, t z_{2}, \ldots, t z_{n}\right), \\
& g_{z}: t \longmapsto g\left(t z_{1}, t z_{2}, \ldots, t z_{n}\right), \\
& h_{z}=f_{z} / g_{z}, \\
& J_{z}: t \longmapsto J\left(t z_{1}, t z_{2}, \ldots, t z_{n}\right)
\end{aligned}
$$


et l'on pose:

$$
h=f / g \text {. }
$$

En prenant $\left(J_{z}\right)_{\lambda}$ comme dans le lemme 7 , on a:

$$
\begin{aligned}
& h_{z}(t)=h\left(t z_{1}, t z_{2}, \ldots, t z_{n}\right), \\
& \left(J_{z}\right)_{\lambda}(t) \leqq J_{\lambda}\left(t z_{1}, t z_{2}, \ldots, t z_{n}\right) .
\end{aligned}
$$

D'après le lemme 7 , il existe $C$ nombre positif non nul indépendent de $z \in \mathbb{C}^{n}$ tel que $|z|=1$ tel que l'on ait:

$$
\begin{aligned}
& \left|h\left(t z_{1}, t z_{2}, \ldots, t z_{n}\right)\right|=\left|h_{z}(t)\right| \\
& \quad \leqq C \exp \left[J_{\lambda}\left(t z_{1}, t z_{2}, \ldots, t z_{n}\right)\right. \\
& \left.\quad+\left\{A^{\prime}(1+\lambda)+B^{\prime}(1+\lambda)\left(\left(\frac{1+\lambda}{\lambda}\right)^{2}-1\right)\right\}|t|\right] .
\end{aligned}
$$

Comme $|t|=|t z|$, on a l'inégalité (20) pour tout $z \in \mathbf{C}^{n}$.

Remarque. D'après le lemme 8 , on peut obtenir le théorème 1 du chapitre II de Malgrange [6] que l'on a utilisé dans la démonstration du théorème 1.

Démonstration de la proposition 7. Soit $T$ une fonctionnelle analytique sur $\mathbf{C}^{n}$ telle que ${ }^{t} P T$ soit appuyée par $\Omega$. Il existe $K$ compact convexe de $\Omega$ tel que ${ }^{t} P T$ soit appuyée par $K$. D'après le théorème de Pólya-EhrenpreisMartineau donné, par exemple, dans Hörmander [3], pour tout nombre positif non nul $\varepsilon$, il existe $C_{\varepsilon}$ nombre positif non nul tel que pour tout $\zeta \in \mathbb{C}^{n}$, on ait:

$$
\left|\mathscr{F}\left({ }^{t} P T\right)(\zeta)\right| \leqq C_{\varepsilon} e^{\varepsilon|\zeta|+J_{K}(\zeta)},
$$

où on se rappelle:

$$
J_{K}(\zeta)=\sup _{z \in K} \operatorname{Re}\langle z, \zeta\rangle
$$

pour tout $\zeta \in \mathbf{C}^{n}$. D'après le lemme 1 , pour tout nombre positif non nul $\delta$, il existe $C_{\delta}^{\prime}$ nombre positif non nul tel que pour tout $\zeta \in \mathbb{C}^{n}$, on ait:

$$
|p(\zeta)| \leqq C_{\delta}^{\prime} e^{\delta|\zeta|}
$$

En changeant les variables, on peut supposer que $p(0) \neq 0$. Dans la notation du lemme 8 , pour tout $\zeta \in \mathbf{C}^{n}$, on a:

$$
\begin{aligned}
\left(J_{K}\right)_{\lambda}(\zeta) & =\sup _{|\eta-\zeta|<\lambda|\zeta|} J_{K}(\eta) \\
& \leqq J_{K}(\zeta)+\lambda|\zeta| M
\end{aligned}
$$


avec $M$ nombre plus grand que la valeur $\sup _{\eta \in K}|\eta|$.

On peut supposer que $M>\varepsilon>0$. Remarquons que l'on a:

$$
\mathscr{F}\left({ }^{t} P T\right)(\zeta)=p(\zeta) \mathscr{F} T(\zeta) .
$$

Alors dans les notations du lemme 8 , en prenant $\lambda=\varepsilon / M$ et $\delta$ plus petit que la valeur

$$
\varepsilon /\left[\left(1+\frac{\varepsilon}{M}\right)\left(\left(\frac{M+\varepsilon}{\varepsilon}\right)^{2}-1\right)\right]
$$

pour tout $\varepsilon$ nombre positif non nul, il existe donc $C$ nombre positif non nul tel que l'on ait:

$$
\begin{aligned}
|\mathscr{F} T(\zeta)| & =\left|\mathscr{F}\left({ }^{t} P T\right)(\zeta) / p(\zeta)\right| \\
& \leqq C e^{5 \varepsilon|\zeta|+J_{k}(\zeta)} .
\end{aligned}
$$

D'après le théorème de Pólya-Ehrenpreis-Martineau, d'où la conclusion.

Comme tout ouvert convexe de $\mathbf{C}^{n}$ est de type de Runge, d'après théorème 2 et proposition 7 , on a le théorème suivant:

Théorème 3. Soient $p\left(\partial_{z}\right)=\sum_{\alpha} a_{\alpha} \partial_{z}^{\alpha}$ un opérateur différentiel non nul à coefficients constants sur $\mathbf{C}^{n}$ représenté par un homomorphisme continu $P$ de $\mathcal{O}$ dans $\mathcal{O}$ et $\Omega$ un ouvert convexe de $\mathbf{C}^{n}$. Alors on a les énoncés suivants (i) et (ii):

(i) Pour toute fonction holomorphe $f$ sur $\Omega$, il existe $u$ fonction holomorphe sur $\Omega$ tel que l'on ait:

$$
p\left(\partial_{z}\right) u=f
$$

dans $\Omega$.

(ii) Toute solution u dans $\Gamma(\Omega, \mathcal{O})$ de l'équation homogène

$$
p\left(\partial_{z}\right) u=0
$$

est approchée dans $\Gamma(\Omega, \mathcal{O})$ par des exponentielle-polynômes satisfaisant à l'équation (22).

Corollaire. Tout homomorphisme continu non nul $P$ de $\mathcal{O}$ dans $\mathcal{O} q u i$ définit un opérateur différentiel à coefficients constants est surjectif.

Remarque. Dans ce mémoire, on a employé la manière de Trèves [8]. 


\section{Références}

[1] Avanissian, V., Fonctions plurisousharmoniques, différences de deux fonctions plurisousharmoniques de type exponentiel, C. R. Acad. Sc., Paris, 252 (1961), 499-500.

[2] Bourbaki, N., Espaces vectoriels topologiques, Hermann, Paris, 1966 et 64.

[3] Hörmander, L., $L^{2}$ estimates and existence theorems for the $\vec{\partial}$ operator, Acta. Math., 113 (1965), 89-152.

[4] Ishimura, R., Faisceaux et équations aux dérivées dans des domaines complexes, thèse de maitrise de la Faculté des Sciences de l'Université de Kyushu, 1978 (en japonais).

[5] Ishimura, R., Homomorphismes du faisceau des germes de fonctions holomorphes dans lui-même et opérateur différentiel, Mem. Fac. Sci. Kyushu Univ., 32 (1978).

[6] Malgrange, B., Existence et approximation des solutions des équations aux dérivées partielles et des équations de convolution. Ann. Inst. Fourier Grenoble, 6 (1955-56), 271-355.

[7] Trèves, F., Linear partial differential equations with constant coefficients, Gordon and Breach, New York, 1966.

[8] — Locally convex spaces and linear partial differential equations, Springer, Berlin, 1967. 
